\title{
Ciclo reprodutivo do mexilhão Perna perna (Linnaeus) \\ (Molusca, Bivalvia) da Lagoa de Itaipu, Niterói, Rio de Janeiro, Brasil
}

\author{
Eliana de Fátima Marques de Mesquita ${ }^{1,2}$ \\ Marta Guimarães de Abreu ${ }^{3}$ \\ Francisco Carlos de Lima ${ }^{1}$
}

\begin{abstract}
Reproductive cycle of Perna perna (Linnaeus) from Itaipu Lagoon, Niterói, Rio de Janeiro, Brazil. The gametogenic aspects of mussel Perna perna (Linnaeus, 1758) from Itaipu Lagoon, Niterói, were studied through November 1998 to October 1999. Microscopic analysis was carried out at low and high dry magnifications, as well as under oil immersion. The animals collected showed variation of size from 4.8 to $9.1 \mathrm{~cm}$ in total length and from 2.0 to $3.9 \mathrm{~cm}$ in total width. Most advanced gonadal stage was detected through May, August and October of 1999 for female specimens. Male advanced gonadal stage occurred in September of the same year. The sex-ratio (M:F) for $P$. perna was 64:52.

KEY WORDS. Perna perna, gonadal cycle, special-staining methods, Itaipu Lagoon, Rio de Janeiro
\end{abstract}

Os aspectos da reprodução e desenvolvimento sexual dos mitilídeos (Mollusca, Bivalvia) têm merecido a atenção de muitos autores. CoE (1943) traçou comentários sobre a diferenciação sexual em vários bivalves, relatando que a unissexualidade é predominante, ocasionalmente ocorrendo o hermafroditismo. Segundo ele, machos e fêmeas distribuem-se de forma igualitária na natureza. Perna perna (Linnaeus, 1758) é molusco de importância alimentar nas regiões onde ocorre em grande quantidade, ou seja, entre os estados do Rio de Janeiro e Santa Catarina (IHERING 1900). Perna perna é considerado o maior dos mitilídeos brasileiros, podendo atingir até $14 \mathrm{~cm}$ de comprimento (KLAPPENBACH 1964). RoJAS (1967) e ROJAS \& MARTINEZ (1967) pesquisaram os aspectos da biologia, principalmente a reprodução e desenvolvimento larval experimental da espécie. EPIFANIO (1967) identificou e descreveu as larvas de P. perna do litoral da Venezuela. UMIJI (1969) estudou a neurosecreção e sua correlação com o ciclo reprodutivo de $P$. perna. LUNETTA (1969) e CARMO \& LUNETTA (1978) desenvolveram estudos relativos ao ciclo reprodutivo de $P$. perna. BERRY (1978) efetuou estudos sobre a reprodução, crescimento e produtividade de $P$. perna na costa africana. ROMERO (1980) verificou os efeitos da salinidade e temperatura sobre embriões e larvas dessa espécie. ABBotT \& DANCE (1986) citam P. perna para a costa oeste da África e Caribe, enquanto Rios (1994) referencia a mesma espécie para Venezuela, toda a costa brasileira até o Uruguai; como também Argentina e sul da África.

1) Departamento de Tecnologia de Alimentos, Universidade Federal Fluminense. Rua Dr. Vital Brazil Filho 64, 24230-340 Niterói, Rio de Janeiro, Brasil. E-mail: eliana@esquadro.com.br

2) Bolsista do CNPq.

3) Bolsista Iniciação Científica FAPERJ/UFF. 
O estudo dos ciclos gametogênicos de bivalves comestíveis, foi enriquecido com a publicação do trabalho de LUNETTA (1969) sobre $P$. perna, quando o autor faz uma análise pormenorizada do ciclo gametogênico, inclusive das modificações bioquímicas da gônada, assim como das influências do meio ambiente.

NARCHI (1976) em sua análise sobre os ciclos gametogênicos de bivalves comestíveis, faz referência ao perigo da extinção de reservas naturais e que a maior coleta de dados para que se possa estabelecer diretrizes eficazes para o cultivo ou para o repovoamento de regiões afetadas, exatamente o que hoje ocorre com a Lagoa de Itaipu, Rio de Janeiro.

A análise histológica das gônadas durante o ciclo sexual é condição básica para que se obtenha parâmetros substanciais para o entendimento do impacto sobre a espécie, levando-se em conta, também, a influência da sazonalidade sobre o mesmo.

A maturação sexual é referenciada como ocorrendo em abril, maio, início de junho e setembro. Os gametas são emitidos durante o ano todo, contudo, durante julho e dezembro a emissão diminui bastante, segundo LunETTA (1969) e BofFI (1979), porém uma certa variação foi observada no local estudado.

\section{MATERIAL E MÉTODOS}

Para a pesquisa foram utilizados 10 exemplares de Perna perna coletados mensalmente na Lagoa de Itaipu junto ao Canal de Camboinhas, Niterói, Rio de Janeiro ( $\left.22^{\circ} 58^{\prime} \mathrm{S}, 43^{\circ} 02^{\prime} \mathrm{W}\right)$, perfazendo uma série de 12 coletas e um total de 120 animais. A primeira coleta, com o auxílio de um formão e/ou faca, ocorreu em novembro de 1998 e a última na segunda quinzena de outubro de 1999. As coletas eram realizadas em vária partes do Canal de Camboinhas, visando uma amostragem mais homogênea da região.

Os espécimes eram fixados, ainda dentro das conchas, porém com as valvas entreabertas, em Bouin por até quatro horas e transferidos para formol a $10 \%$ por 24 horas. As valvas foram medidas e separadas as partes moles. Foi efetuada a secção transversal, incluindo divertículo digestivo, manto, brânquias, gônadas e pé. Depois de desidratados, os tecidos foram embebidos em parafina, seccionados a $5 \mu \mathrm{m}$ em corte transversal, e corados pela técnica de HE, descrita por HowARD et al. (1983). Procedia-se a análise macro e microscópica e medição dos oócitos em estádios avançados da oogênese. Para a medição dos oócitos utilizou-se a técnica da régua milimetrada cuja unidade é o micrômetro, levando a um resultado direto na leitura sob objetiva de imersão. Técnicas especiais de coloração, PAS, DPAS, van Gieson e Giemsa, segundo BEHMER et al. (1976), foram aplicadas para evidenciação, respectivamente, de glicogênio, células mucíparas, fibras colagênicas, e protozoários.

\section{RESULTADOS E DISCUSSÃO}

Os animais coletados na Lagoa de Itaipu apresentaram uma variação de tamanho de 4,8 a $9,1 \mathrm{~cm}$ de comprimento e 2,0 a 3,9 $\mathrm{cm}$ de largura. A literatura registra que $P$. perna apresenta um tamanho médio de $5-8 \mathrm{~cm}$ de comprimento por 3-4 $\mathrm{cm}$ de largura e 2-3 $\mathrm{cm}$ de espessura (BOFFI 1979). 
O sex-ratio (M:F) encontrado foi de 64:52 demonstrando que os machos encontram-se ligeiramente em maior número neste local em particular. COE (1943) já havia feito referência à distribuição igualitária de machos e fêmeas na natureza.

Preferiu-se seguir a classificação do estádio de desenvolvimento reprodutivo segundo Powell et al. (1993) que abrange os estádios 1 a 8, porque os estádios estão melhor definidos e oferecendo maior clareza na leitura das lâminas do que a de LUNETTA (1969). No período de novembro de 1998 a outubro de 1999 os estádios reprodutivos 1 a 5 e 8 foram observados nas fêmeas da espécie $P$. perna; já nos machos foram encontrados os estádios reprodutivos de 2 a 7 , porém não sendo observados os estádios reprodutivos de 6 a 7 nas fêmeas e 8 nos machos (Fig. 1, Tab. I).

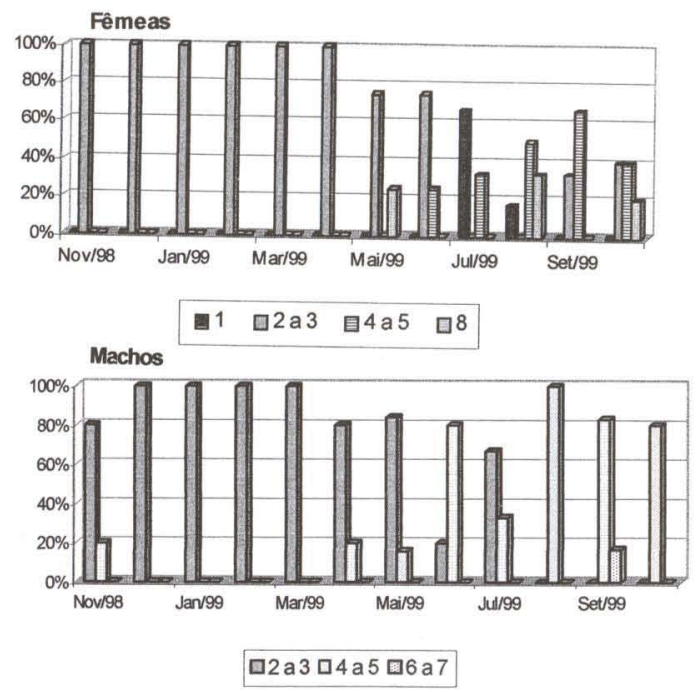

Fig. 1. Estádios reprodutivos em Perna perna.

Tabela I. Percentagem dos diferentes estádios reprodutivos de Perna perna.

\begin{tabular}{|c|c|c|c|c|c|c|c|c|c|c|}
\hline \multirow{2}{*}{ Mês / Ano } & \multicolumn{5}{|c|}{ Fêmeas (\%) } & \multicolumn{5}{|c|}{ Machos (\%) } \\
\hline & 1 & $2-3$ & $4-5$ & $6-7$ & 8 & 1 & $2-3$ & $4-5$ & $6-7$ & 8 \\
\hline Novembro/1999 & 0 & 100 & 0 & 0 & 0 & 0 & 80 & 20 & 0 & 0 \\
\hline Dezembro/1999 & 0 & 100 & 0 & 0 & 0 & 0 & 100 & 0 & 0 & 0 \\
\hline Janeiro/1999 & 0 & 100 & 0 & 0 & 0 & 0 & 100 & 0 & 0 & 0 \\
\hline Fevereiro/1999 & 0 & 100 & 0 & 0 & 0 & 0 & 100 & 0 & 0 & 0 \\
\hline Março/1999 & 0 & 100 & 0 & 0 & 0 & 0 & 100 & 0 & 0 & 0 \\
\hline Abril/1999 & 0 & 100 & 0 & 0 & 0 & 0 & 80 & 20 & 0 & 0 \\
\hline Maio/1999 & 0 & 75 & 0 & 0 & 25 & 0 & 84 & 16 & 0 & 0 \\
\hline Junho/1999 & 0 & 75 & 25 & 0 & 0 & 0 & 20 & 80 & 0 & 0 \\
\hline Julho/1999 & 67 & 0 & 33 & 0 & 0 & 0 & 67 & 33 & 0 & 0 \\
\hline Agosto/1999 & 17 & 0 & 50 & 0 & 33 & 0 & 0 & 100 & 0 & 0 \\
\hline Setembro/1999 & 0 & 30 & 67 & 0 & 0 & 0 & 0 & 83 & 17 & 0 \\
\hline Outubro/1999 & 0 & 40 & 40 & 0 & 20 & 0 & 0 & 80 & 0 & 0 \\
\hline
\end{tabular}



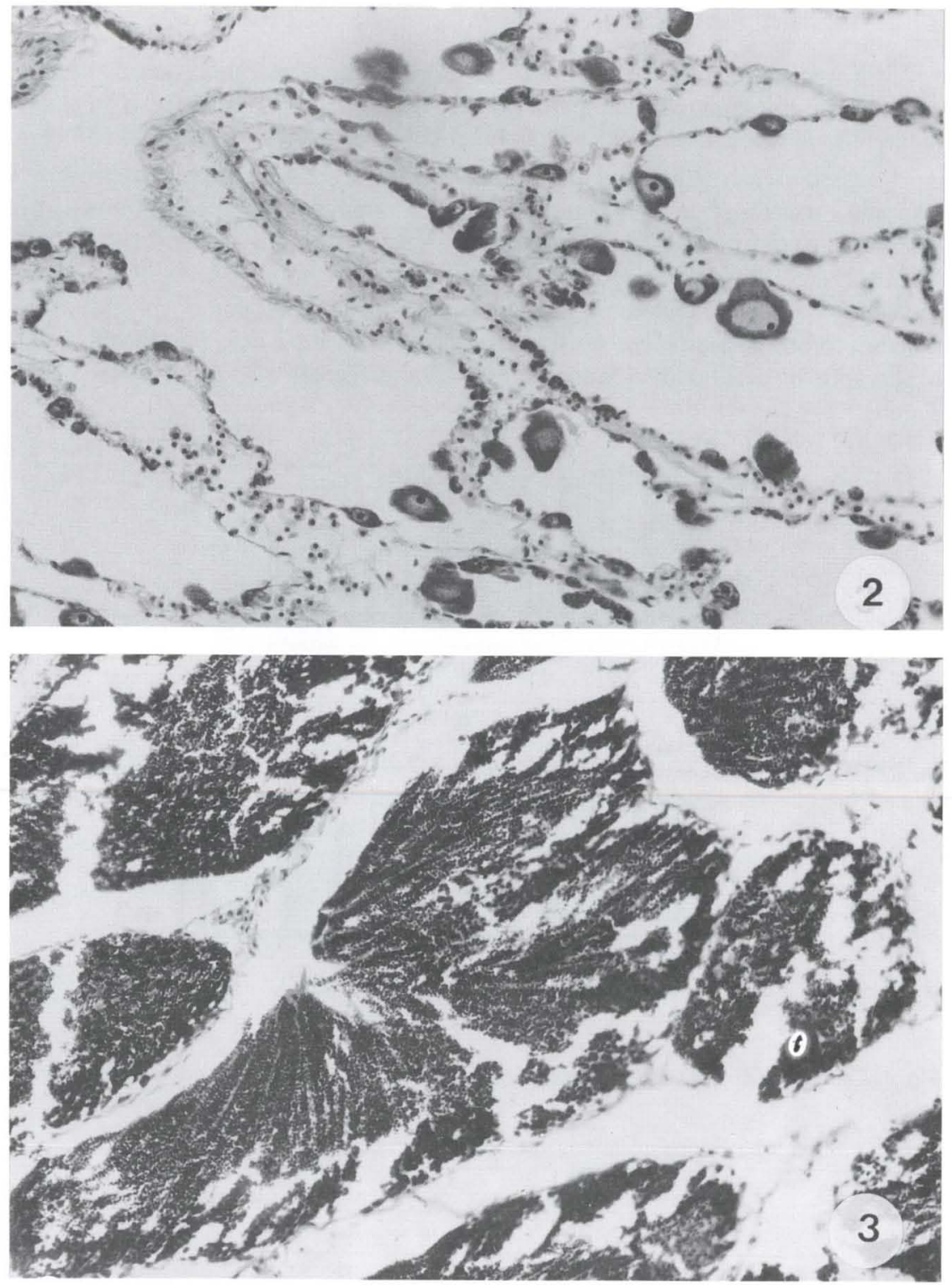

Figs 2-3. Corte transversal da gônada de Perna perna. (2) Feminina, no estádio 8; (4) masculina nos estádios 6-7. Coloração: HE.

O estádio reprodutivo mais avançado de fêmeas de $P$. perna, estádio 8 , mostrou-se nos meses de maio, agosto e outubro de 1999 nas fêmeas com as seguintes percentagens, respectivamente: $25 \%, 33 \%, 20 \%$ (Fig. 2). Já os estádios reprodutivos mais avançados dos machos da mesma espécie, estágios de 6 a 7 , ficaram situados no mês de setembro de 1999 com a seguinte percentagem: 17\% 
(Fig. 3). Ficando, assim, em desacordo com os estudos de LUNETTA (1969) e Boffi (1979) que observaram que os gametas eram emitidos durante o ano todo. Este fato não foi observado com a mesma espécie na Lagoa de Itaipu.

O tecido epitelial do manto de $P$. perna mostrou-se PAS positivo demonstrando a presença de células mucíparas produtoras de mucopolissacarídeos neutros. A membrana basal do epitélio do trato digestivo apresentou-se DPAS positiva demostrando a presença de glicogênio. Foi possível observar a disposição do tecido conjuntivo e do tecido muscular, pelo van Gieson, notando-se que os exemplares da espécie em estudo apresentavam menor quantidade de tecido muscular e maior quantidade de tecido conjuntivo.

Projetos sobre esse tema estão sendo desenvolvidos na Lagoa de Itaipu, visando um monitoramento histológico constante e avaliação do impacto de fatores ambientais e antropogênicos sobre esta e outras espécies de interesse econômico da região.

\section{REFERÊNCIAS BIBLIOGRÁFICAS}

Aвbott, R.T.; P. DAnCE. 1988. Compedium of Seashells. A color guide to more than $\mathbf{4 , 2 0 0}$ of the world's marine shells. New York, E.P. Dutton Inc., $411 \mathrm{p}$.

Behmer, O.A.; E.M.C. Tolosa; A.G. Freitas Neto. 1976. Manual de técnicas para histologia normal e patológica. São Paulo, Edart, 255p.

BERRY, P.F. 1978. Reproduction, growth and production in the mussel Perna perna (L.), on the east coast of South Africa. Oc. Res. Inst. Invest. Rep. 48: 1-28.

Boffi, A.V. 1979. Moluscos brasileiros de interesse médico e econômico. São Paulo, Ed. HUcitec, $182 \mathrm{p}$.

CARMo, T.M.S. \& J.E. LunetTA. 1978. Changes in the lipid level of Perna perna (Linné, 1758) (Mollusca

- Bivalvia), related to sex and sexual stages. Bol. Fisiol. Animal, São Paulo, 2: 49-62.

CoE, W.R. 1943. Sexual differentiation in mollusks. I. Pelecypods. Quart. Jour. Rev. Biol. 18 (2): 154-164.

EPIFANIO, R.M. 1967. Identificacion y descripcion de la larva veliconcha y dissoconcha del mejillon comestible Perna perna (L) del Oriente de Venezuela. Ser. Recursos Explotacion Pesqueros 1 (3): $95-113$.

Howard, D.C. \& C.S. SMITH. 1983. Histological Techniques for Marine Bivalve Mollusks. NOAA Technical Memorandum NMFS-F/NEC-25. Massachusetts, Woods Hole, 97p.

IherING, H. von. 1900. On the south American Species of Mytilidae. Proc. Malac. Soc. Lond. 4: 84-98. KLAPPENBACH, M.A. 1964. Lista preliminar de los Mytilidae brasileños com claves para su determinación y notas sobre su distribuicion. An. Acad. Brasil. Ciênc. 37 (Supl.): 327-352.

LUNETTA, J.E. 1969. Fisiologia da reprodução de mexilhões (Mytilus perna) (Mollusca: Lamellibranchia)

Bol. Fac. Filos. Ciên. Letr. Univ. São Paulo, 324 Zool. Biol. Mar. (N.S.) 26: 33-111.

NARCHI, W. 1976. A importância do conhecimento dos ciclos gametogênicos de bivalves comestiveis. Seminários de Biologia Marinha. São Paulo, 09 a 15 de dezembro de 1975. An. Acad. Brasil. Ciênc. 47 (Supl.): 133-134.

Powell, E.N.; E.A. Wilson-Ormond \& K-S. ChOI. 1993. Gonadal Analysis - Crassostrea virginica. NOAA Technical Memorandum NOS ORCA 71 National Status and Trends Program for Marine Environmental Quality, Vol. 2, Comprehensive Descriptions of Complementary Measurements, p. II.55-II.61.

Rios, E.C. 1994. Seashells of Brazil. Rio Grande, Editora FURG, $2^{a}$ ed., 368p.

RoJAs, A.V. 1967. Observaciones experimentales sobre algunos aspectos biológicos del mejillón comestible (Perna perna Retzius). Lagena 14: 14. 
Rojas, A.V. \& E.R. Martinez. 1967. Reproducción y desarollo larval esperimental del mejillón comestible de Venezuela, Perna perna (Linnaeus, 1758). Bol. Inst. Oceanogr., Cumana, 6: 266-284.

Romero, S.M.B. 1980. Características comportamentais e morfológicas dos estágios larvais de Perna perna (Lamellibranchia: Mytilidae) obtidos em laboratório. Bol. Fisiol. Anim. Univ. São Paulo 4: 45-52,

UmiJ, S. 1969. Neurosecreção em Mytilus perna (Molusco Lamelibrânquio) Bol. Fac. Filos. Ciên. Letr. Univ. São Paulo, 324 Zool. Biol. Mar. (N.S.), 26: 181-254.

Recebido em 27.IV.2000; aceito em 12.VI.2001. 